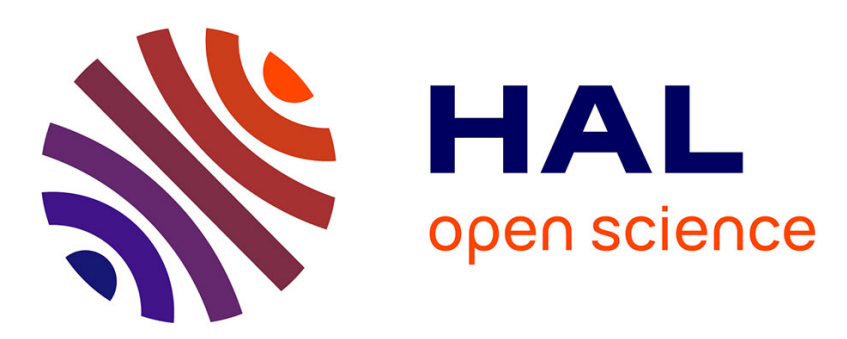

\title{
Atomic-scale modelling of elastic and failure properties of clays
}

\author{
G. Hantal, Laurent Brochard, H. Laubie, E. Ebrahimi, R-J-M. Pellenq, F.J. \\ Ulm, Benoit Coasne
}

\section{- To cite this version:}

G. Hantal, Laurent Brochard, H. Laubie, E. Ebrahimi, R-J-M. Pellenq, et al.. Atomic-scale modelling of elastic and failure properties of clays. Molecular Physics, 2014, 112 (9-10), pp.1294-1305. hal01021110

\section{HAL Id: hal-01021110 https://hal.science/hal-01021110}

Submitted on 20 Jun 2018

HAL is a multi-disciplinary open access archive for the deposit and dissemination of scientific research documents, whether they are published or not. The documents may come from teaching and research institutions in France or abroad, or from public or private research centers.
L'archive ouverte pluridisciplinaire HAL, est destinée au dépôt et à la diffusion de documents scientifiques de niveau recherche, publiés ou non, émanant des établissements d'enseignement et de recherche français ou étrangers, des laboratoires publics ou privés. 
1 Atomic-scale modeling of elastic and failure properties of clays

3 György Hantal, ${ }^{1}$ Laurent Brochard, ${ }^{2}$ Hadrien Laubie, ${ }^{1}$ Davoud Ebrahimi, ${ }^{1}$ Roland J.-M.

4 Pellenq, ${ }^{1,3,4}$ Franz-Josef Ulm, ${ }^{1,4}$ and Benoit Coasne ${ }^{1,4,}$

$6 \quad{ }^{1}$ Department of Civil and Environmental Engineering, Massachusetts Institute of Technology, 77

7 Massachusetts Avenue, Cambridge, MA 02139, United States

$8 \quad{ }^{2}$ Université Paris-Est, Laboratoire Navier (UMR 8205), CNRS, ENPC, IFSTTAR, 77455 Marne-la-

$9 \quad$ Vallée, France

$10 \quad{ }^{3}$ CINaM-CNRS, Campus de Luminy, 13288 Marseille cedex 09, France

$11{ }^{4}$ Multiscale Material Science for Energy and Environment $<$ MSE $>^{2}$, The joint CNRS-MIT

12 Laboratory, 77 Massachusetts Avenue, Cambridge MA 02139, United States

13

14

15

16

·To whom correspondence should be made: coasne@mit.edu 


\section{Abstract}

2 The elastic and failure properties of a typical clay, illite, are investigated by means of molecular simulation.

3 We employ a reactive (ReaxFF) as well as a non-reactive (ClayFF) force field to assess the elastic properties

4 of the clay. As far as the failure properties are concerned, ReaxFF was used throughout the study, however

5 some calculations were also performed with ClayFF. A crack parallel to the clay layers is found to have low

6 fracture resistance (equivalent fracture toughness $K_{I c}=0.09 \mathrm{MPa} \cdot \mathrm{m}^{1 / 2}$ ) when submitted to a tensile loading

7 perpendicular to the crack (mode I). The nanoscale mechanism of both yield and fracture failures is

8 decohesion in the interlayer space, and the critical energy release rate characterizes both failures. In contrast,

9 under shear loading (mode II), the nanoscale failure mechanism is a stick-slip between clay layers. No fracture

10 propagation is observed as the clay layers slide on top of each other. The low fracture resistance in mode I and

11 the stick-slip failure in mode II are both the consequence of the lack of chemical bonds between clay layers

12 where the cohesion is provided by electrostatic interactions only. We also consider the mode I loading of a

13 crack perpendicular to the clay layers and find that, in this case, the material exhibit strain softening as the

14 result of the clay layers breaking one after the other. In this orientation illite displays a much higher fracture

15 resistance $\left(0.61 \mathrm{MPa} \cdot \mathrm{m}^{1 / 2}\right)$ due to the breaking of chemical bonds involved when fracturing in this direction.

16 This work, which provides a description of the failure properties of clays at the microscopic scale, is a first

17 step needed to describe the failure of clays at a larger scale where the polycrystalline distribution of clay

18 grains is a key parameter that must be taken into account. 
2 Oil and gas shale rocks have received particular attention in the past decades as source rocks for fossil fuel production. (Vandenbroucke \& Largeau, 2007) Due to the very low permeability of shale formations, the common recovery technique is based on stimulating the rock by hydraulic fracturing. In particular, clay-rich shales seem difficult to operate. Britt and Schoeffler report that shale with clay content higher than $40 \%$ are seldom producing enough hydrocarbons to ensure profitability. (Britt \& Schoeffler, 2009)Various hypotheses were proposed to explain those difficulties, e.g., low intrinsic permeability, incapability to activate natural fractures, high in-situ stress, or swelling. Clearly, understanding the fundamental elastic and fracture properties of clay-rich shale rocks is of key importance to operate in a more efficient and eco-friendly way.

11 Among major components in shales, clays are layered aluminosilicates that are also found in sediments, soils, and argillaceous rocks. (Bergaya, Then, \& Lagaly, 2006)Clays have been used as raw material in pottery and construction for millennia, but they have also been gaining importance in new technological fields over the past decades. Modern applications of clays often rely on their swelling properties as they can uptake water or selectively organic molecules and intercalate them between clay layers. Clays have also found applications due to their large specific surface area which is responsible for a number of their unique physical and chemical properties. Moreover, the fact that the adsorption and cation exchange capacity of clay minerals can be controlled with the $\mathrm{pH}$ makes clays ideal candidates for a broad range of applications. Recently, they have also been used as natural or artificial barriers in radioactive waste depositories due to their very low permeability and long-term healing capacity. Clay-polymer complexes have also been used in inorganicreinforced nanocomposites in fields ranging from aerospace systems to commodity plastics. (Vaia, 2002)

Owing to the numerous applications above, the structural and mechanical properties of clays have received significant attention. However, because of their high affinity for water and their fine-grained polycrystalline nature, measuring these properties is challenging. Indeed, clays usually appear in the form of aggregates of 
little crystals of a size smaller than $2 \mu \mathrm{m}$. Further difficulties are related to the fact that clay grains are often mixed with other minerals, which renders obtaining reliable structural data, other than unit cell parameters, also problematic. Even though their anisotropy is well known, very often only isotropic elastic properties of clays are reported due to the limitations of experimental techniques in describing micro-scale mechanical properties of clay grains. (Vanorio, Prasad, \& Nur, 2003), (Faust \& Knittle, 1994)

Molecular modeling provides a viable alternative to experimental techniques as it allows one to probe material properties on the molecular scale. As a result, there have been several computational studies dedicated to the investigation of the anisotropic elastic properties of clays. Heinz reported cleavage energies for different clay minerals and clay/surfactant composites based on classical molecular dynamics simulations. (Heinz, 2006) This author found good agreement with available experimental measurements. Soto et al. was the first to use first-principles calculations to determine the elasticity tensor of a layered silicate, kaolinite. (Sato, Ono, Johnston, \& A., 2005) Similar first-principles calculations were performed by Zartman et al. and Militzer et al. to obtain elasticity parameters of different clay minerals including pyrophyllite, montmorillonite, kaolinite, and muscovite as a function of the number and the distribution of isomorphic substitutions. (Zartman, Liu, Akdim, Pachter, \& Heinz, 2010) (Militzer, Wenk, Stackhouse, \& Stixrude, 2011) Teich-McGoldrick et al. used the classical ClayFF force field to determine the pressure and temperature dependence of the elastic properties of muscovite and found better agreement with experiments than earlier first-principles calculations. (Teich-McGoldrick, Greathouse, \& Cygan, 2012) Ebrahimi et al. also carried out classical molecular simulations with ClayFF to determine the elastic properties of Na-montmorillonite as a function of water uptake. (Ebrahimi, Pellenq, \& Whittle, 2012)

The focus of this paper is on the elastic and failure properties of illite, one of the most common clay minerals in Earth's crust. (Bergaya, Then, \& Lagaly, 2006) In particular, we aim to determine on the molecular scale its elasticity tensor as well as its resistance to fracture and failure mechanisms under mechanical loading. We first determine the elastic properties of illite with two different molecular force fields, and compare their ability to 


\section{2. Computational details}

\section{$6 \quad$ 2.1. Clay model: illite}

7 Illite is one of the most commonly found clay minerals in soils. (Bergaya, Then, \& Lagaly, 2006) It is also a 8 typical constituent of sedimentary rocks, especially shales. Illite, or more precisely the few distinct clay

predict the elasticity tensor. We also determine the failure properties of illite for different crack orientations based on an empirical reactive force field. To the best of our knowledge, this study is the first to determine fracture properties of clays by means of a reactive molecular force field.

minerals that are referred to as illite, constitute a sub-group in the dioctahedral 2:1 phyllosilicate family. For the sake of simplicity, in what follows, we will use the term illite to refer to one typical composition. The structure of illite is illustrated in Figure 1a. Illite is defined as a non-swelling clay with exclusively potassium ions in the interlayer space. Pyrophyllite is considered the reference structure for dioctahedral 2:1 phyllosilicates as it forms charge-neutral clay layers where all tetrahedral $(\mathrm{T})$ and octahedral $(\mathrm{O})$ positions in the structure are filled with $\mathrm{Si}$ and $\mathrm{Al}$, respectively. Illite differs from pyrophyllite in that (i) about one tetrahedral silicon position per unit cell is filled with aluminum and (ii) aluminum in one octahedral position of every second unit cell is substituted in an isomorphous way, with other metal atoms. Such metal atoms can be either of lower valence than $\mathrm{Al}$ (e.g., $\mathrm{Mg}(\mathrm{II})$ or $\mathrm{Fe}(\mathrm{II})$ ) or, less typically, of the same valence (e.g., $\mathrm{Fe}(\mathrm{III})$ ). In case $\mathrm{Al}$ is replaced with a metal atom having a lower valence, the resulting charge defect is compensated with potassium ions. The typical charge of clay layers in illite varies between $-1 e$ and $-1.8 e$ per unit cell depending on the number of isomorphic substitutions of lower valence. In this study we used the composition given by Meunier et al., $\mathrm{K}_{(\mathrm{x}+\mathrm{y})}\left[\mathrm{Al}_{(4-\mathrm{y})} \mathrm{Mg}_{\mathrm{y}}\right]\left(\mathrm{Si}_{(8-\mathrm{x})} \mathrm{Al}_{\mathrm{x}} \mathrm{O}_{20}\right)(\mathrm{OH})_{4}$, (Meunier \& Velde, 2004) and chose $\mathrm{x}=1$ and $\mathrm{y}$ $=0.5$ to have an overall clay charge equal to $-1.5 e$ per unit cell.

The starting configuration for illite used in our work is the pyrophyllite unit cell optimized from highaccuracy quantum chemistry calculations by Refson et al. (Refson, Park, \& Sposito, 2003) In the simulation 
cell, $\mathrm{Si}(\mathrm{IV}) \rightarrow \mathrm{Al}(\mathrm{III})$ isomorphic substitutions were made in the top or bottom (T) layers of every replicated

2 unit cell together with $\mathrm{Al}(\mathrm{III}) \rightarrow \mathrm{Mg}(\mathrm{II})$ substitutions in the (O) layer of every second unit cell. We ensured

3 that no $\mathrm{O}$ atom had more than one substituted metal neighbor in order to satisfy the Lowenstein rule (Bergaya,

4 Then, \& Lagaly, 2006)). We also verified that an equal number of substitutions were made in the top and

5 bottom (T) layers to avoid highly asymmetrical charge distributions and, hence, large electrostatic fields in the

6 system. Potassium ions were distributed randomly in the clay interlayer space to counterbalance the

7 electrostatic charges induced by the isomorphic substitutions in the layers.

$\mathbf{a}$

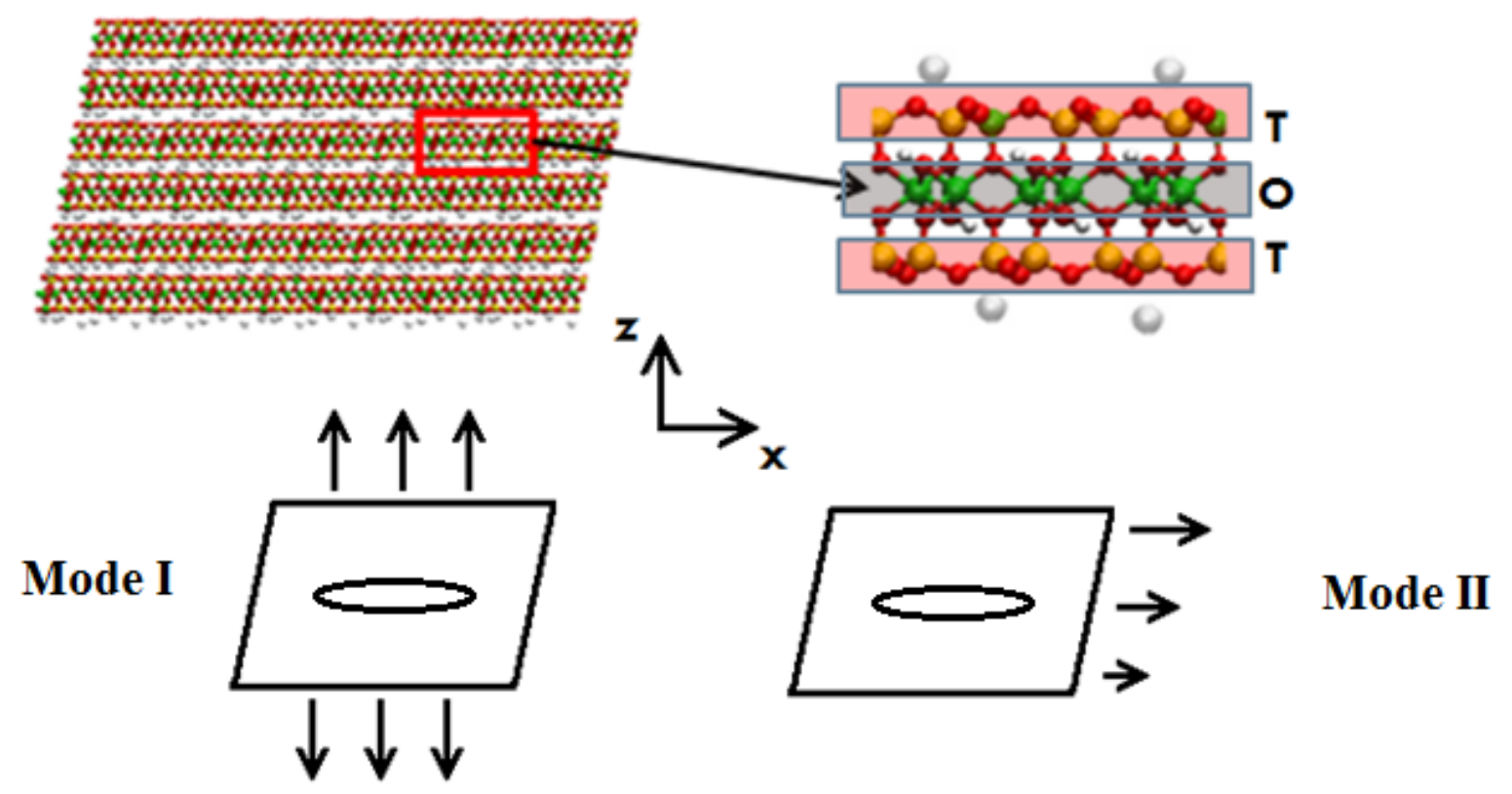

Figure 1. (a) Structure of illite whose chemical formula is $\mathrm{K}_{1.5}\left[\mathrm{Al}_{3.5} \mathrm{Mg}_{0.5}\right]\left(\mathrm{Si}_{7} \mathrm{AlO}_{20}\right) \mathrm{OH}_{4}$. Yellow, green, red, white and grey spheres correspond to silicon, aluminum, oxygen, hydrogen and potassium, respectively. Illite is made up of the stacking of tetrahedral $(\mathrm{T})$ and octahedral $(\mathrm{O})$ layers. About one $(\mathrm{T})$ silicon position per unit cell is filled with aluminum and aluminum in one $(\mathrm{O})$ position of every second unit cell is substituted in an isomorphous way with other metal atoms. (b) Schematic illustration of the different mechanical loadings applied to the system with an elliptical crack; while a tensile loading perpendicular to the plane of the crack (xy plane here) acts on the system upon mode I ("opening mode"), a shear loading is applied parallel to the plane of the crack upon mode II ("sliding mode"). The crack is infinitely long in the y direction and propagation is expected to occur in the xy plane. 


\section{$1 \quad$ 2.2. Elastic properties}

2 There are different ways to obtain the elastic properties of solids from molecular simulation. A widely used

3 approach is based on applying a series of small strains to the system and calculating the resulting stress tensor.

4 If the deformation is small enough and the system remains in the linear elastic regime, the stress-strain curve

5 can be fitted with a straight line whose slope provides the stiffness parameters. The advantage of this method

6 is that is can be used at any finite temperatures, however, its application is often not possible as the result may

7 exhibit strain rate dependence. Another approach to determine the elastic properties of solids is to estimate the curvature of the potential energy surface about a minimum energy configuration. Indeed, the components of

9 the symmetrical stiffness tensor $C_{i j}$ (in Voigt notation) can be written as the second derivative of the energy 10 density with respect to the strains $\varepsilon_{i}$ and $\varepsilon_{j}$ :

$$
C_{i j}=\frac{1}{V} \frac{\partial^{2} U}{\partial \varepsilon_{i} \partial \varepsilon_{j}},
$$

where $V$ is the volume of the simulation cell and $U$ is the potential energy. $\varepsilon_{i}$ and $\varepsilon_{j}(i, j=1 \ldots 6)$ represent the components of the second order strain tensor in Voigt notation. Here we use a Cartesian coordinate frame where direction 3 is perpendicular to the clay layers. For simple potential energy functions the second derivatives of the energy are often available analytically. In such a case the stiffness tensor can readily be determined at any point of the potential energy surface. If the functional form is too complicated, as in the case of the ReaxFF potential used in the present work, the 21 independent elements of the stiffness tensor can be calculated by applying linear combinations of all elementary deformations and performing an energy minimization of the resulting structures. The obtained energy profiles are then fitted with a parabola which provides a numerical estimate of the curvature of the potential energy surface about the energy minimum.

21 Such a curvature is then used to estimate $C_{i j}$ from Eq. 1.Note that strictly speaking this method provides elastic parameters at $0 \mathrm{~K}$. If the entropic contribution to the free energy is small $C_{i j}$ values determined at $0 \mathrm{~K}$ are not very different from values at finite temperatures. This is often the case for minerals with high melting point, like clays. However this assumption does not hold for solids of low melting point (e.g. polymers). 
1

2

3

4

\subsection{Failure properties}

Fracture failure refers to the failure of materials where a flaw, like a micro-crack, is responsible for the occurrence of a crack in a localized region of a body. By contrast, yield failure refers to the failure of flawless materials. In this work, we assessed both failure properties by simulating illite with and without an initial micro-crack. According to fracture mechanics, at the continuum scale, the resistance of a material to fracture is characterized by a few material properties. (Anderson, 2005) In the simplest formulation, valid for linear elastic materials under the assumption of small scale yielding, a single property is sufficient, which is either the stress intensity $K$ or the energy release rate $G$. When the stress intensity reaches a critical value, called fracture toughness $K_{c}$, the crack propagates (or, equivalently, when the energy release rate reaches a critical value $G_{c}$ ). The usual assumption is that $K_{c}$ and $G_{c}$ are material properties. Regarding yield, the failure is characterized by the maximum stress conditions, the yield strength or yield surface, that the flawless material can sustain. In the general case, yield strength and fracture resistance are not correlated. In this work, we focus on the particular fracture and yield properties of illite for which a peculiar yield-fracture relation is possible because of the layered structure.

In the present work, we use a thermodynamic route to derive the critical energy release rate, in which the variation of free energy of the system is estimated from molecular simulation (for a detailed description of the method the reader is advised to refer to Ref (Brochard, Hantal, Laubie, Pellenq, \& Ulm)). In isothermal conditions, the thermodynamic state of a deformable solid can be described by the applied mechanical loading and, if there is, the crack area. Upon loading, the mechanical work provided to the system and stored in recoverable energy is released when fracture propagation occurs. Our goal is to determine by means of molecular simulations the released energy when the fracture propagates, i.e. upon increasing the surface $A_{\text {crack }}$ of an existing crack by a quantity $\Delta A_{\text {crack }}$. The balance of Helmholtz free energy $F$ of the system under displacement loading is:

$$
d F=d P+G_{C} d A_{\text {crack }} \text { where } P=\int_{V}\left(\int_{0}^{\varepsilon(\mathrm{r})} \underline{\underline{\sigma}}(\varepsilon): d \underline{\underline{\varepsilon}}\right) \mathrm{dr}
$$


2 The change in the free energy $\mathrm{d} F$ includes the variation in the mechanical energy $\mathrm{d} P$ and the energy released

3 by propagating the crack $G_{C} \mathrm{~d} A_{\text {crack }}$. The mechanical energy can be locally evaluated by integrating the stress

$4 \underline{\underline{\sigma}}(\varepsilon)$ over the local strain $\mathrm{d} \underline{\underline{\varepsilon}}(\mathrm{r})$ at any point $\mathbf{r}$. A second integration over the whole volume of the system $V$

5 yields the total mechanical energy stored in the system. In these equations the double-underlined letters

6 correspond to second-order tensorial quantities. In isothermal equilibrium conditions, the free energy is

7 minimum $\mathrm{d} F=0$, which leads to the usual definition of the critical energy release rate $G_{c}$ :

$$
G_{C}=\left(\frac{-\partial P}{\partial A_{\text {crack }}}\right)_{T, \text { loading }},
$$

9 where the derivative is at constant temperature and loading. Akin to real experiments, we apply in molecular simulations a load to the system and measure its mechanical response. In our study, this is done by imposing

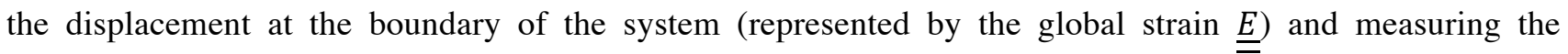
resulting stress $\underline{\underline{\Sigma}}$ at the boundaries of the sample.The integration of $\underline{\underline{\Sigma}}$ with respect to $\underline{\underline{E}}$ provides an estimate of the variation of free energy of the system per unit volume. If, at the end of the simulation, the system is in the same loading state as in the beginning, the mechanical energy has been totally released by crack surface creation so that the integration of the stress-strain curve yields the critical energy release rate $G_{C}$ :

$$
G_{C}=V \frac{\int_{0}^{E}{ }_{\text {final }} \underline{\underline{\Sigma}}(E): \mathrm{d} \underline{\underline{E}}}{\int_{0}^{E} \text { final } \mathrm{d} A_{\text {crack }}},
$$

17 where $\int_{0}^{E_{\text {final }}} \mathrm{d} A_{\text {crack }}=\Delta A_{\text {crack }}$ is the area of crack created. It is possible to relate the energy release to the 18 stress intensity factor through Irwin's formula. In a general case, for the linear combination of all three 19 loading modes the generalized Irwin formula can be used:

$$
G=\pi^{t}\{K\} \cdot[H] \cdot\{K\}
$$

21 where $\{K\}=\left(K^{I}, K^{I I}, K^{I I I}\right)$ is a vector containing the stress intensity factors of the three loading modes $(\mathrm{I}=$ 22 "opening", II = "sliding", and III = "tearing" modes). $[H]$ is a $3 \times 3$ matrix that depends on the elastic properties of the material. ${ }^{\mathrm{t}}\{\}$ denotes the transpose of a vector. While finding the $[H]$ matrix is usually not 
straightforward, its determination can be simplified in case of simple loadings or material symmetries. Here we will use the solution derived for plane-strain conditions, under which all strains in the material are restricted to lie in a plane. If a strain acts in the xy plane, only $\varepsilon_{\mathrm{xx}}, \varepsilon_{\mathrm{yy}}$ and $\varepsilon_{\mathrm{xy}}$ are non-zero. In the general case of plane strains, there always exists a coordinate frame in which the strain tensor has only 3 non-zero elements, which reduces the $3 \mathrm{D}$ problem to a $2 \mathrm{D}$ one.

\subsection{Evaluation of the crack surface area}

As shown in the previous section, evaluating the fracture energy $G_{c}$ requires the determination of the area of the fracture created upon releasing the mechanical energy (see Eq. 4). This is far from being a trivial task in molecular simulations due to the fractal-like character of atomic surfaces which is a consequence of the discontinuous nature of matter at this scale. All molecular surface analysis methods necessarily have an adjustable parameter that characterizes its sensitivity to the size of discontinuities and, hence, the resolution of the given method. There is thus no unique way to evaluate surface area in molecular simulations .Among the various surface analysis methods (Lee \& Richards, 1971), (Connolly, 1983), (Jorge, Jedlovszky, \& Cordeiro, 2010), we chose to apply a methodology similar to that yielding the solvent-accessible surface, (Lee \& Richards, 1971) also known as the specific surface. In this algorithm a probe atom of a certain size rolls over all surface atoms. The specific surface is defined as the set of points visited by the center of the probe during rolling. In our study, this surface is estimated by a Monte Carlo procedure; (Bhattacharya, Coasne, Hung, \& Gubbins, 2009) segments are generated in the system with random position and direction that may intersect the surface. The specific surface area $\left(S_{S S}\right)$ of the void-wall interface per unit volume is then calculated as

$$
S_{S S}=2 \frac{\Sigma_{i=1}^{N} P_{i}}{\sum_{i=1}^{N} l_{i}}
$$

where $p_{i}$ is the number of intersections of the segments $i \quad(=1 \ldots N)$ of length $l_{i}$ with the interface. The algorithm works as follows. A random location is picked in the system, which is the initial point of the probe segment. If a probe sphere of radius $r_{p}$ can be inserted at this point without overlapping with any atom, this point is considered as belonging to the "void". In contrast, if the probe sphere overlaps with any of the atoms, 
the point is considered to belong to the "wall". The next point analyzed of the segment is at a distance $\Delta l$ from

2 the previous point in a direction chosen randomly (but kept constant for the same segment). Again, this point

3 is characterized as belonging either to the "void" or to the "wall" by checking whether a probe sphere of

4 radius $r_{p}$ could be accommodated at this point without overlapping with the wall atoms. An intersection (i.e., a

5 surface point) is detected when two consecutive points on a given segment belong to different domains (either wall or void). If the procedure is run for a large number of segments, it provides a representative sample of surface points being located at distances $d \in\left[r_{p}, r_{p}+\Delta l\right]$ from the wall atoms. The choice of the maximum length of the segment as well as of $\Delta l$ affects the efficiency of the procedure but not its sensitivity. The latter is controlled by the radius of the probe sphere $r_{p}$. There is no unique choice of this quantity, rather a reasonably defined range of possible values. Our criterion for setting $r_{p}$ was to allow the crack opening to be detected in the clay interlayer, i.e., it was such that when the system is intact (there is no fracture in the system) the probe sphere is large enough not to fit into the clay interlayer. However it fits in (and hence detects surface creation) once the crack starts to propagate. This led us to set $r_{p}=3.2 \AA$. Note that during the analysis, molecular fragments detached from illite as well as $\mathrm{K}^{+}$ions were excluded. Figure 2 illustrates the way the surface analysis method above works together with a crack and an example of a set of sampled surface points.

a)

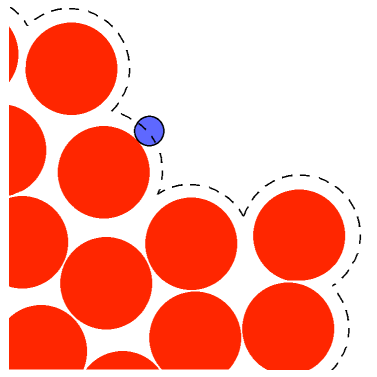

b)

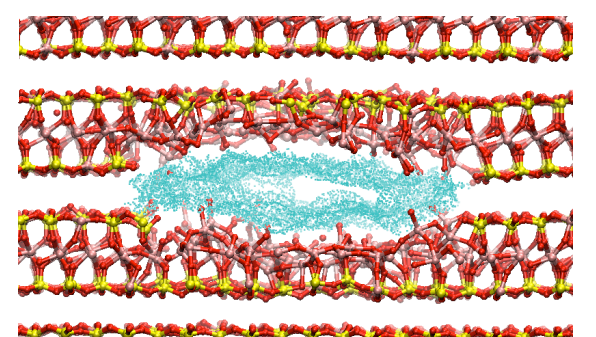

Figure 2.(a) Illustration of the surface (dashed line) accessible for the blue probe sphere of radius $r_{p}$ in a system of the red particles (b) A representative sample of the surface points (grey) obtained in a crack by a 
Monte Carlo type algorithm (see text). Yellow, purple and red spheres correspond to silicon, aluminum and oxygen atoms (hydrogen atoms are not shown for better visibility).

\subsection{Molecular Dynamics simulations}

5 Propagation of fracture in solids involves breaking and formation of chemical bonds. At the molecular scale, these complex processes can be efficiently simulated with a reactive force field. In this study we chose to use the ReaxFF reactive force field, (van Duin, Dasgupta, Lorant, \& Goddard, 2001), (van Duin, Strachan, Stewman, Zhang, Xu, \& Goddard, 2003) which is one of the largest existing bond order potentials with available parameterizations for various kinds of molecular system. We used the parameter set which was developed by Pitman and van Duin (Pitman \& van Duin, 2012)to study the reactivity of water confined between zeolite and smectite. As this set does not contain parameters for potassium ions, the original parameters for sodium ions were modified by replacing those that describe basic physical properties such as the atomic mass and sigma bond covalent radius, as well as non-bonded interaction parameters described by the Morse potential. These latter parameters were obtained to best fit the Lennard-Jones potential of K ions as described by the ClayFF potential. (Cygan, Liang, \& Kalinichev, 2004) These changes only affect the intermolecular part of the interactions. Since potassium is not expected to participate in covalent bond

17 formation and only acts as a counterion, these modifications are thought to be satisfactory and relevant. As a test of our results, some simulations were also performed with the non-reactive ClayFF potential, which is known to accurately reproduce structural data of various clay phases. This is due to the fact that most interatomic interactions are treated in ClayFF as non-bonded interactions which lends a large flexibility to the force field. ClayFF was also successfully used to simulate transport in clays. (Botan, Marry, Rotenberg, Turq, \& Noetinger, 2013) (Wang, Kalinichev, \& Kirkpatrick, 2006)

24 Molecular dynamics simulations were carried out with the LAMMPS simulation program package at $300 \mathrm{~K}$. 25 (Lammps: Molecular Dynamics Simulator, http://lammps.sandia.gov) The time step for the integration of the 
molecular equations of motion was chosen equal to $0.1 \mathrm{fs}$ in the case of ReaxFF and $1 \mathrm{fs}$ in the case of ClayFF.

2 After setting up the initial configuration, the system was first energy minimized. A series of deformations was

3 then applied; each deformation was followed by an energy minimization and then by a molecular dynamics

4 simulation at constant volume and temperature for $10 \mathrm{ps}$. Equilibrated configurations were sampled and saved

5 for further analysis. Periodic boundary conditions were used in the three directions $x, y$, and $z$. The elements of

6 the stress tensor were calculated with the virial expression and averaged for every deformation step: (Allen \&

$7 \quad$ Tildesley, 1989)

$$
\Sigma_{\alpha \beta}=-\frac{1}{a_{x} b_{y} c_{z}}\left\langle\sum_{i=1}^{N} \frac{p_{i, \alpha} p_{i, \beta}}{m_{i}}+\sum_{i=1}^{N} r_{i \alpha} f_{i, \beta}\right\rangle,
$$

where $\mathbf{a}, \mathbf{b}$, and $\mathbf{c}$ denote the three box vectors and the subscripts $x, y$, and, $z$ refer to vectorial components along the three directions in the Cartesian coordinate frame. $\alpha$ and $\beta$ represent any of these components. The index $i(=1, . ., N)$ runs over all $N$ atoms in the system. $m_{i}, \mathbf{p}_{i}$, and, $\mathbf{r}_{i}$ correspond to the mass, momentum, and position of atom $i$, respectively. $\mathbf{f}_{i}$ is the force acting on atom $i$.

\section{Results and discussion}

\subsection{Elastic properties of illite}

We used the numerical method (introduced in Section 2.3) to obtain the stiffness tensor of illite with the ReaxFF forcefield (presented in Table 1). Figure 3 illustrates the fitting procedure of the obtained energy profiles against a parabolic function, which allows estimating for each type of deformation the stiffness constant $C_{i j}$ using Eq. (6). The elasticity tensor of illite was also computed with the ClayFF forcefield (shown in Table 2) using the GULP 3.1 program package (Gale, 2002) which provides the full stiffness tensor from the analytical derivatives of the intermolecular interactions after a thorough energy minimization of the system. All values in Tables 1 and 2 were averaged over 5 equilibrium configurations. Both matrices are positive definite, which ensures that the energy minimized systems correspond to stable structures. 


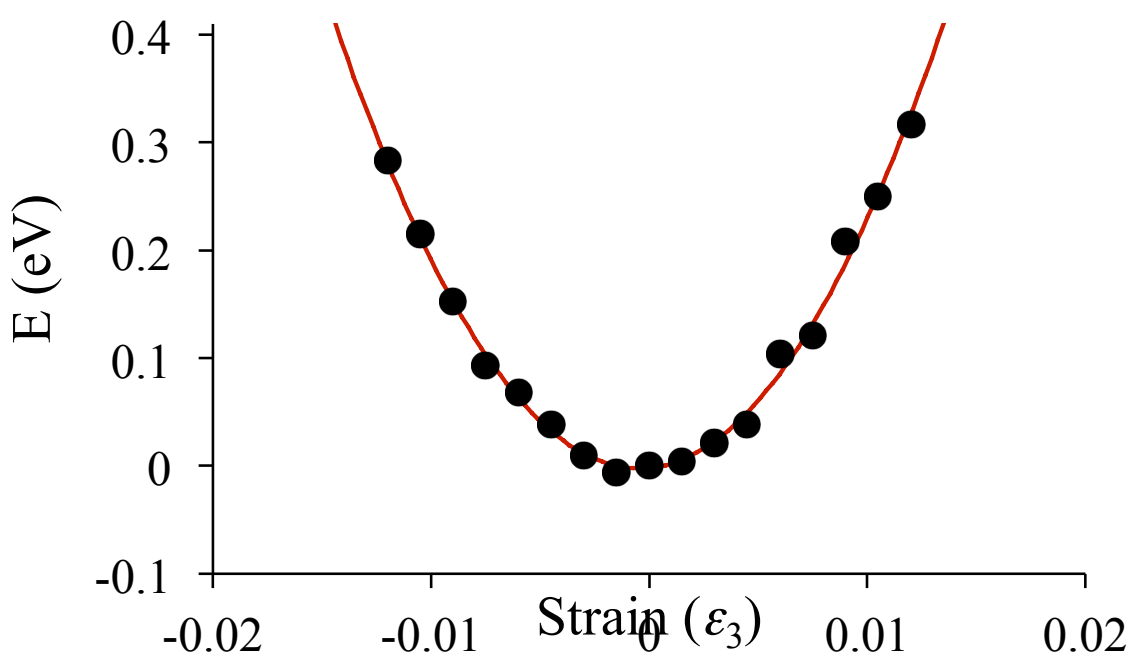

2 Figure 3. Determination of the the stiffness parameters $C_{i j}$ from the curvature of the potential energy surface.

3 In this specific example, the deformation $\varepsilon_{3}$ is along the direction 3 . These data were obtained at $300 \mathrm{~K}$ using 4 ReaxFF.

5

6 Table 1. Stiffness tensor of illite in Voigt notation as obtained with ReaxFF. Note that the tensor is shown

7 here as an upper triangular matrix due to symmetry reasons $\left(C_{i j}=C_{j i}\right)$. The shaded fields in the table show the 8 elements that are expected to be different from 0 for a transversely isotropic material (see text).

\begin{tabular}{|c|c|c|c|c|c|c|}
\hline $\mathrm{C}_{\mathrm{ij}} / \mathrm{GPa}$ & 1 & 2 & 3 & 4 & 5 & 6 \\
\hline 1 & $216 \pm 5$ & $76 \pm 9$ & $29 \pm 4$ & $-4 \pm 3$ & $-21 \pm 2$ & $-3 \pm 2$ \\
\hline 2 & & $209 \pm 4$ & $29 \pm 5$ & $-1 \pm 3$ & $-9 \pm 2$ & $0 \pm 2$ \\
\hline 3 & & & $93 \pm 1$ & $-7 \pm 1$ & $-23 \pm 1$ & $-4 \pm 1$ \\
\hline 4 & & & & $4.7 \pm 0.6$ & $0 \pm 1$ & $-1.5 \pm 0.4$ \\
\hline 5 & & & & & $10.8 \pm 0.4$ & $4 \pm 3$ \\
\hline 6 & & & & & & $14 \pm 2$ \\
\hline
\end{tabular}

9 
1 Table 2. Stiffness tensor of illite in Voigt notation as obtained with ClayFF. Note that the tensor is shown

2 here as an upper triangular matrix due to symmetry reasons $\left(C_{i j}=C_{j i}\right)$. The shaded fields in the table show the

3 elements that are expected to be different from 0 for a transversely isotropic material (see text).

\begin{tabular}{|c|c|c|c|c|c|c|}
\hline $\mathrm{C}_{\mathrm{i} j} / \mathrm{GPa}$ & 1 & 2 & 3 & 4 & 5 & 6 \\
\hline 1 & $292.5 \pm 0.5$ & $128.3 \pm 0.4$ & $16.67 \pm 0.08$ & $1.0 \pm 0.3$ & $5.5 \pm 0.6$ & $-17.0 \pm 0.1$ \\
\hline 2 & & $274.2 \pm 0.4$ & $15.97 \pm 0.04$ & $0.1 \pm 0.7$ & $2.0 \pm 0.3$ & $1.7 \pm 0.1$ \\
\hline 3 & & & $48.9 \pm 0.1$ & $0.37 \pm 0.03$ & $2.89 \pm 0.06$ & $0.87 \pm 0.01$ \\
\hline 4 & & & & $8.99 \pm 0.02$ & - & $0.8 \pm 0.2$ \\
\hline 5 & & & & & $0.71 \pm 0.02$ & \\
\hline 6 & & & & & $9.82 \pm 0.04$ & $-0.1 \pm 0.2$ \\
\hline
\end{tabular}

5 It is useful to consider the random polycrystal properties, i.e., the macroscopic elastic moduli, which can be obtained in the framework of the Voigt-Reuss-Hill (VRH) approximation classically used in mineralogy. In

7 this approach one simply takes the average of the values obtained based on the definitions given by Voigt

8 (using the stiffness tensor) and those defined by Reuss (using the compliance tensor which is the inverse of

9 the stiffness tensor, $\mathbf{S}=\mathbf{C}^{-1}$ ). Tables 3 summarizes the VRH polycrystal properties of illite computed with

10 ReaxFF and ClayFF.

12 Table 3. Elastic moduli of illite as obtained with the ReaxFF and ClayFF forcefields.

\begin{tabular}{lcc}
\hline \hline & ReaxFF & ClayFF \\
\hline Bulk modulus / GPa & $52 \pm 6$ & $73.66 \pm 0.08$ \\
\hline Shear modulus / GPa & $18 \pm 2$ & $35.17 \pm 0.03$ \\
\hline Speed of sound: $v_{s} / \mathrm{km} / \mathrm{s}$ & $2.6 \pm 0.1$ & $3.517 \pm 0.001$ \\
\hline Speed of sound: $v_{p} / \mathrm{km} / \mathrm{s}$ & $5.4 \pm 0.3$ & $6.511 \pm 0.003$ \\
\hline Young's moduli / GPa & 1 & $110 \pm 51$ \\
\hline
\end{tabular}




\begin{tabular}{lccc}
\hline 2 & $146 \pm 44$ & $215.4 \pm 0.2$ \\
\cline { 2 - 4 } & 3 & $24 \pm 16$ & $46.9 \pm 0.2$ \\
\hline \hline
\end{tabular}

Tables 1-3 reveal that, in general, ReaxFF predicts a more compliant mechanical behavior than ClayFF. Values in column 3 of Tables 1 and 2 are exceptions since ReaxFF seem to predict stiffer behavior along the direction perpendicular to the clay layers (direction 3). It is worth noting that, considering the stiffness tensor $\left(\mathrm{C}_{11}>\mathrm{C}_{22}>\mathrm{C}_{33}\right.$, see Table 1), the trend in the Young's moduli calculated with ReaxFF $\left(E_{2}>E_{1}>E_{3}\right.$, see Table 3) is counterintuitive. However, this is not observed in the case of ClayFF (cf. Tables 2 and 3). Note also that the error computed with ReaxFF is two orders of magnitude larger than that of ClayFF.

9 We also examined the anisotropy of the elasticity tensor. It is common practice to consider clays transversely isotropic (TI) materials. For such materials the elastic properties show a specific rotational symmetry: in planes perpendicular to the axis of the rotational symmetry, elastic properties are isotropic (in Figure 1, this is the $\mathrm{z}$ axis). Due to its high symmetry, the elastic properties of a TI material obey the following relationships: $\mathrm{C}_{11}=\mathrm{C}_{22}, \mathrm{C}_{13}=\mathrm{C}_{23}, \mathrm{C}_{44}=\mathrm{C}_{55}$ and $\mathrm{C}_{66}=\left(\mathrm{C}_{11}-\mathrm{C}_{12}\right) / 2$. As a result, a TI material has only five independent stiffness constants out of $21: \mathrm{C}_{11}, \mathrm{C}_{12}, \mathrm{C}_{13}, \mathrm{C}_{33}, \mathrm{C}_{44}$ and all other elements are zero (note that similar symmetry relations hold for the compliance tensor). A comparison of the obtained elasticity tensors of our models to that of an ideal transversely isotropic material provides a test of the reliability of the computed tensors. Considering the data obtained for illite with ReaxFF (Table 1), this comparison reveals that $\mathrm{C}_{11}$ and $\mathrm{C}_{22}$ are indeed very close as they only differ by $2 \%$. Considering the uncertainty over the results, $\mathrm{C}_{13}$ and $\mathrm{C}_{23}$ can also

19 be considered equal. However, $\mathrm{C}_{55}$ is roughly twice larger than $\mathrm{C}_{44}$, while $\mathrm{C}_{66}$ is 5 times smaller than $\left(\mathrm{C}_{11^{-}}\right.$ $20 \mathrm{C}_{12}$ )/2. In addition, $\mathrm{C}_{34}, \mathrm{C}_{15}, \mathrm{C}_{25}$, and $\mathrm{C}_{35}$ are found to be significantly different from 0 . The data obtained with 21 ClayFF (Table 2) displays a much better agreement with what is expected for a TI material: $\mathrm{C}_{11}$ and $\mathrm{C}_{22}$ are not strictly equal but their difference is only about $6 \% . \mathrm{C}_{13}$ and $\mathrm{C}_{23}$ are found to differ by about $4 \%$ while the departure of $\mathrm{C}_{44}$ from $\mathrm{C}_{55}$ amounts to $8 \%$. $\mathrm{C}_{66}$ deviates from $\left(\mathrm{C}_{11}-\mathrm{C}_{12}\right) / 2$ by $10 \%$. The rest of the values (white cells in Table 2) are all almost zero, except for the element $\mathrm{C}_{16}$. 
2 Further comparison of the elasticity tensors obtained using ReaxFF and ClayFF can be achieved by measuring their distance from the tensor of a TI material using an appropriate distance metric. Despite its simplicity and elegance, the well-known Euclidean metric sometimes leads to ambiguities in the interpretation of results.

5 Here we chose to use the Riemannian metric as it avoids such ambiguities. (Moakher, 2006)The Riemannian 6 metric between two positive definite $n \times n$ matrices, $\mathbf{A}$ and $\mathbf{B}$, is defined as:

$$
d_{\mathrm{R}}(\mathrm{A}, \mathrm{B})=\left\|\ln \left(B^{1 / 2} A^{-1} B^{1 / 2}\right)\right\|=\left[\sum_{i=1}^{n} \ln ^{2} \lambda_{i}\right]^{1 / 2},
$$

where $\lambda_{i}$ are the eigenvalues of $\mathbf{A}^{-1} \mathbf{B}$. For each computed stiffness tensor, a reference TI matrix was generated and the distance of this reference to the original tensor was calculated using the Riemannian metric. The inverse of such a reference matrix $\mathbf{S}^{\text {ref }}$ is determined based on the inverse of the computed stiffness matrices

11 (i.e., the compliance matrix $\mathbf{S}): \mathrm{S}_{11}{ }^{\text {ref }}=\left(\mathrm{S}_{11}+\mathrm{S}_{22}\right) / 2, \mathrm{~S}_{33}{ }^{\text {ref }}=\mathrm{S}_{33}, \mathrm{~S}_{44}{ }^{\text {ref }}=\left(\mathrm{S}_{44}+\mathrm{S}_{55}\right) / 2, \mathrm{~S}_{13}{ }^{\text {ref }}=\left(\mathrm{S}_{13}+\mathrm{S}_{23}\right) / 2$, and $\mathrm{S}_{12}{ }^{\text {ref }}$ $12=\mathrm{S}_{11}\left(\mathrm{~S}_{12} / \mathrm{S}_{11}+\mathrm{S}_{12} / \mathrm{S}_{22}\right) / 2$. The rest of the elements of the reference matrix is set to 0 . Such an analysis reveals that the Riemannian distance of the tensors calculated with ReaxFF and ClayFF to their reference matrix is 2.66 and 0.37 , respectively. In other words, ClayFF predicts elastic properties that are closer to those of a TI material. To quantify the difference between the two computed matrices and the reference TI matrices, it is also possible to calculate the error one would make by using the reference TI matrix instead of the original one when calculating fracture toughness. For cracks oriented parallel with the plane of transverse isotropy (cracks in the 001 plane, see below), this comparison yields $108.5 \%$ error for ReaxFF but only $2.6 \%$ for ClayFF.

Depending on the applied molecular force field, elastic constants, especially in-plane constants, have been found to differ significantly. (Zartman, Liu, Akdim, Pachter, \& Heinz, 2010) Although desirable, the comparison of our results with experimental data is prohibited due to the lack of experimental values. Therefore, we use the above qualitative comparisons to discriminate between the two sets of data: we conclude that our molecular simulations with ClayFF give a reliable description of the elastic properties of 
illite, due to the close resemblance of the ClayFF elasticity tensor to that of a TI material. In contrast, ReaxFF

2 gives a poor description of illite as a TI material. We will thus use, in the rest of this study, the reliable

3 elasticity tensor computed with the ClayFF potential and combine with fracture energies obtained from

4 ReaxFF when reactivity needs to be taken into account.

\subsection{Failure parallel to clay layers}

To study both yield failure and fracture failure, we simulate bulk illite both with and without an existing crack. As the clay layers are not bonded covalently, the basal (001) plane is an ideal plane for cleavage for

9 both fracture failure and yield failure. Thus, we focus our investigation on the possible relationship between 10 fracture and yield. Figure $1 \mathrm{~b}$ illustrates a crack lying in the xy plane (i.e., the basal plane). In the ideal case the 11 thickness of the crack along the $\mathrm{z}$ direction must be negligible compared to its dimensions in the other 12 directions. In our simulations, elliptical cracks with high aspect ratios $(\approx 4-5$, i.e., the ratio of the major and minor axes of the ellipse) were created in the interlayer region. We consider two crack sizes of lengths 32.5 ands $53 \AA$ and an intact structure (i.e., without a pre-existing crack). For the intact structure, we consider three different system sizes besides the 'original' size: a system $50 \%$ longer or $50 \%$ thicker. Such we could assess the dependence of the results to crack size and system size.

\subsubsection{Mode I loading}

The calculated stress-strain curves display the same qualitative behavior upon mode I loading, which confirms that it is independent of the studied crack and system size. As an illustration, we present the stress-strain curve obtained in a system without an initiated crack in Figure 4. This figure shows that illite exhibits very low resistance to mechanical loading characterized by low values of the critical strain $(<0.05)$ and critical stress $(<$

$232.5 \mathrm{GPa}$ ). It is also seen that the material is in the linear regime at low loadings, which is followed by a 24 nonlinear strain hardening while approaching the point where the material fails and the stress drops to zero. 25 Irrespective of the presence of an initial crack in the system, all the systems fail by cleavage in the interlayer 
space. Accordingly, the nanoscale mechanism of fracture failure and yield failure are qualitatively similar. As

2 shown in Eq.(3), one has to estimate the crack surface area in order to estimate the fracture energy $G_{c}$ from the

3 integral of the strain-stress curves. To evaluate the crack area, the method introduced in Section 2.4 was

4 applied. In Figure 4, besides the stress-strain curve, the crack area in the same system is also plotted as a

5 function of the strain imposed. These data show that the chosen probe size is appropriate to describe fracture

6 propagation as the change in the crack surface area (i) is negligible in the elastic regime and (ii) increases

7 abruptly as the fracture starts propagating. For both intact and initially cracked systems, the energy released is

8 proportional to the area of uncracked material. This proves that fracture failure and yield failure originate from

9 the same mechanism: a decohesion in the interlayer space which can be characterized by an energy released

10 per unit area, that is, by an energy release rate (Eq. 4). The nanoscale heterogeneity of the clay leads to a

11 peculiar relationship between yield and fracture failures. Usually, in perfectly homogenous material, yield

12 would distribute over the volume with a homogenous strain field and the energy released would be

13 proportional to the volume; while fracture concentrates the stresses and strains in a plane. The average fracture

14 energy $G_{c}$ obtained by averaging over the five different systems considered in this work, is $G_{c}=0.22 \pm 0.04$ $\mathrm{J} / \mathrm{m}^{2}$. This value is quite low owing to the lack of chemical bonds between the clay layers.

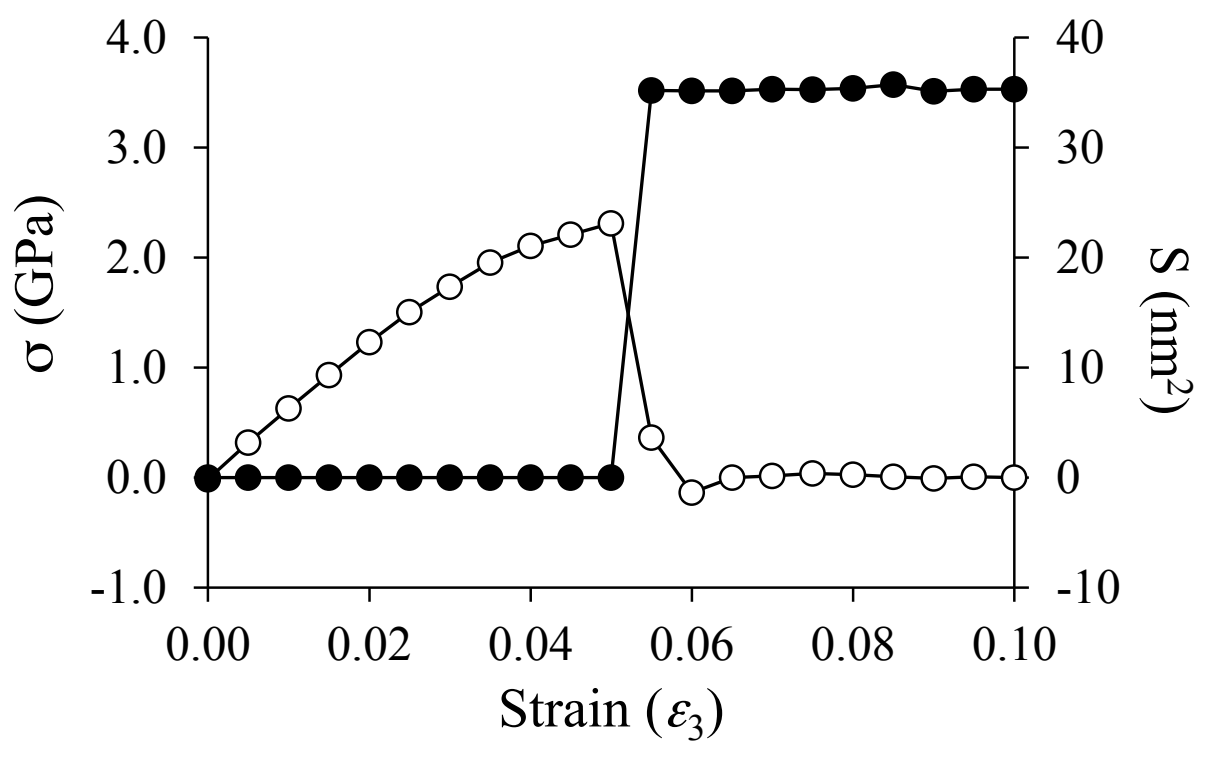

18 Figure 4.Stress $\sigma$ (black empty circles, left axis) and crack surface area $S$ (black filled circles, right axis) 19 obtained in simulating mode I loading of the interlayer space as a function of the imposed strain $\varepsilon_{3}$. These data 
were obtained for the smallest system without a pre-existing crack (the other systems display similar qualitative results). The crack area was estimated using a probe $r_{p}=3.2 \AA$ (see Section 2.4).

4 It is worth mentioning that we found that systems thinner in the $\mathrm{z}$ direction than our thinnest system produced unphysical stress oscillations after crack propagation. This is unrealistic as the system, once already broken, shows increasing stress (and then also stress of opposite sign) upon further strain increase although there is no more cohesion between the two broken parts of the fracture. Moreover, for such a small system size, the clay layers were found to oscillate before and after crack propagation. We believe that this is most likely due to the small size of the system considered.

As pointed out above, the pre-existence of a crack in the system is not needed for a fracture to propagate in the direction parallel to the clay layers. This means that the molecular simulations above can be repeated with a non-reactive force field such as ClayFF in order to confirm the failure behavior of clays. Note that this is only true when the system does not contain an initial crack as a reactive forcefield is otherwise needed to account for possible charge deficiencies as well as surface reconstruction. The obtained stress-strain curve calculated with ClayFF is shown in Figure 5. This curve shows the same brittle mechanism as that obtained with ReaxFF. However, illite simulated with ClayFF reaches higher strains before failing $(\varepsilon=0.1)$. Moreover, the stress-strain curve obtained with ClayFF yields a fracture energy $G_{C}=0.34 \mathrm{~J} / \mathrm{m}^{2}$, which is about $50 \%$ larger than the value obtained with ReaxFF. This is probably due to the lack of long range corrections of the electrostatic interaction in the ReaxFF potential which leads to weaker cohesion in illite.

As shown in Section 3.1, the stiffness tensor computed with ClayFF can be very well approximated with that of a TI material. Owing to this symmetry of the system, it is possible to provide a simple expression for $H_{l l}$ (see Eq.(5)),the matrix element needed to convert energy release rate to stress intensity (all other elements are irrelevant in pure mode I).The original expression by Sih et al. (Sih, Paris, \& Irwin, 1964) has been rewritten in terms of the stiffness constants. (Laubie, 2013)This equation reads for plane-strain cases as: 


$$
H_{11}=\frac{1}{2 \pi} \sqrt{\frac{C_{11}}{C_{11} C_{33}-C_{13}^{2}}\left(\frac{1}{C_{44}}+\frac{2}{C_{13}+\sqrt{C_{11} C_{33}}}\right)}
$$

3 Using the stiffness matrix components in Table 2 one obtains fracture toughnesses of 0.12 and $0.097 \mathrm{MPa} \cdot \mathrm{m}^{1 / 2}$

4 for ClayFF and ReaxFF, respectively. These fracture toughnesses are very low compared to typical values of

5 metal oxides $\left(\sim 1 \mathrm{MPa} \cdot \mathrm{m}^{1 / 2}\right)$, (Brochard, Hantal, Laubie, Pellenq, \& Ulm), (Spray, 2010)which is the result of

6 the absence of chemical bonds between the clay layers where the cohesion is provided by electrostatic forces

7 only. Indeed, as will be seen in Section 3.4, a much larger fracture toughness is obtained when a crack

8 perpendicular to clay layers is investigated i.e., when chemical bond breaking is involved in the fracture

9 propagation.

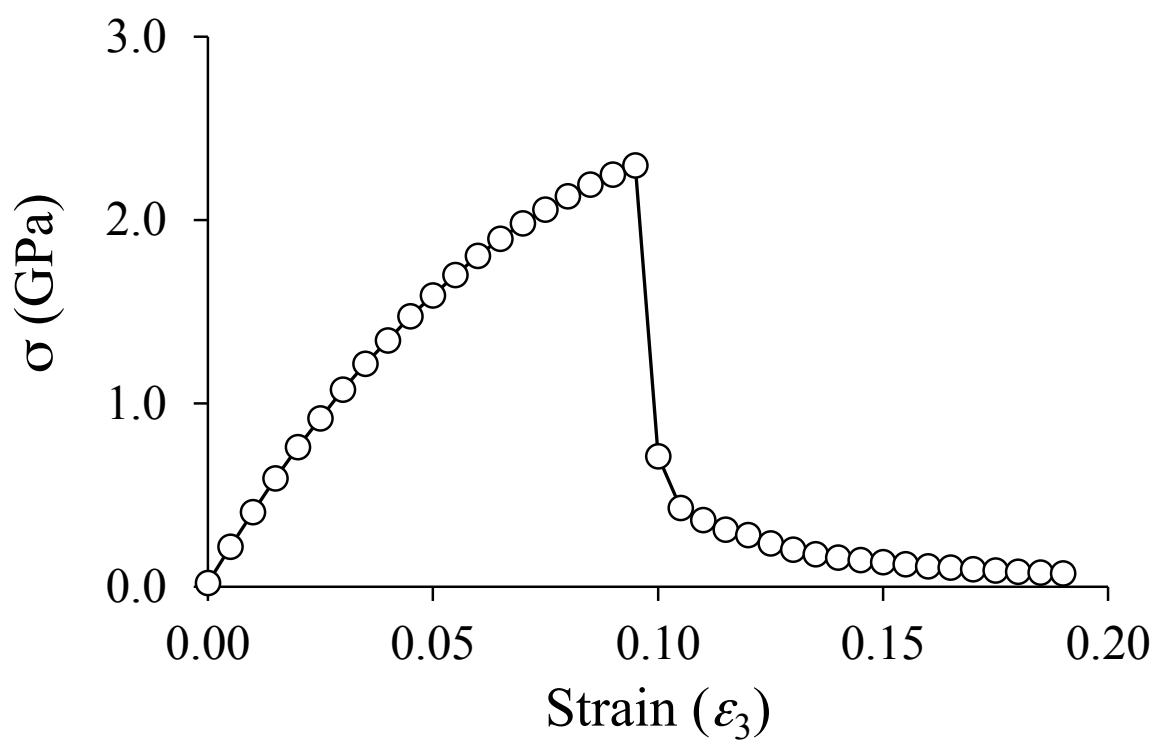

11 Figure 5. Stress-strain curve obtained with ClayFF when illite is loaded in mode I in a direction parallel to the 12 clay layers.

\subsubsection{Mode II loading}

Again, all stress-strain curves obtained for different crack and system sizes are found to display the same behavior. As a result, we present in Figure 6 only one stress-strain curve obtained upon mode II loading parallel to the clay layers. The system in mode II loading exhibits a behavior that is radically different from what was observed for mode I loading. After an initial stable increase of the stress upon increasing the load, an instability occurs and the stress sharply drops. This drop is, however, followed by a new stable stress 
1 increase until the stress drops again. These successive stable and unstable regimes alternate upon increasing

2 the shear strain. This is characteristic of a failure with stick-slip. For the systems with crack, crack does not

3 propagate. The system does not fracture, but it releases mechanical energy as the clay layers slide on the top

4 of each other. At the macroscale, we expect the local stick-slips to be averaged which would led to an

5 apparent plastic behavior. Similar failure mechanism was identified by Zartman et al. in molecular simulations

6 upon applying shear deformation in the same direction to similar clays. (Zartman, Liu, Akdim, Pachter, \&

7 Heinz, 2010)

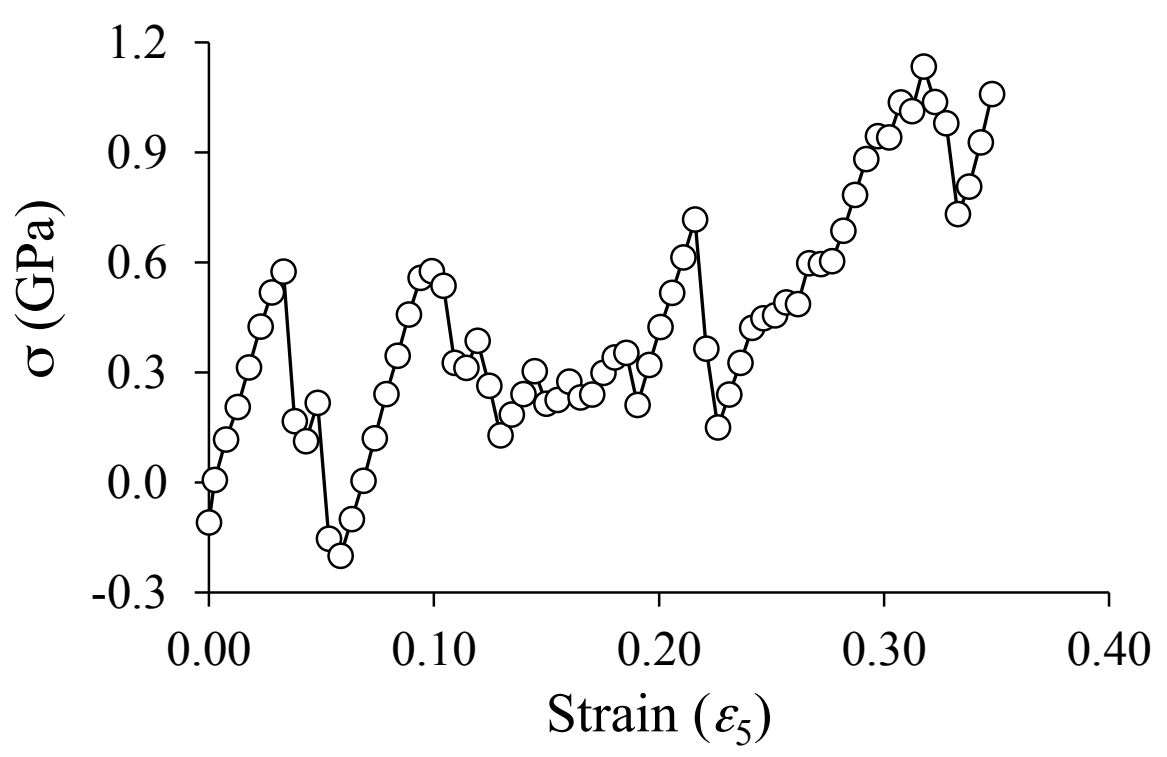

9 Figure 6.Stress-strain curve upon mode II loading of a crack parallel to the clay layers in illite. The observed behavior suggests that the fracture does not propagate because the mechanical energy gets dissipated as the clay layers slide on the top of each other. This stick-slip failure of illite upon mode II applied in the direction parallel to the clay layers can be understood by considering the layered structure of illite. The observed mechanism is confirmed by visualizing the molecular configurations saved in our simulations. This is again due to the absence of chemical bonds in the clay interlayer where only electrostatic interaction provides the cohesion in the structure. This means that

17 at the microscopic scale, illite (and presumably other clays) with this crack orientation would not produce fracture propagation when loaded in mode II. The mode II loading of the material along clay layers was also performed with ClayFF. The stress-strain curve computed with this potential (Figure 7) shows the same 
mechanism, but the curve displays a much greater regularity than those obtained with ReaxFF. The stick-slip mechanism is almost periodic. It is worth noticing that the stress value at which the slip of clay layers occur is considerably higher with ClayFF than the highest value reached with ReaxFF (1.9 GPa vs $0.8 \mathrm{GPa}$, see Figures 6 and 7). This can be related to the missing long range correction in ReaxFF.

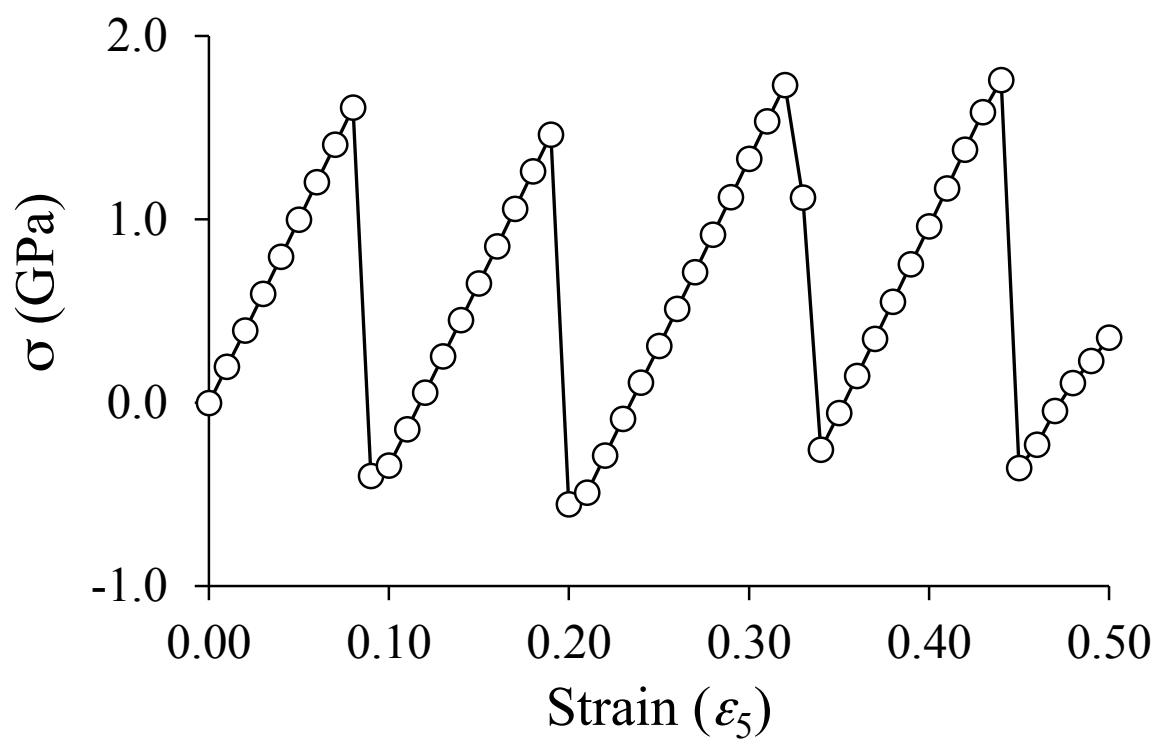

6

\subsection{Fracture orthogonal to clay layers}

In addition to cracks parallel to the bedding plane of clay layers, we also considered cracks perpendicular to the 001 plane (i.e., to the clay layers). In this section we investigate the mode I loading of such cracks. Given that fracture propagation in this direction requires breaking chemical bonds, the molecular simulations can only be performed (i) with a pre-existing crack and (ii) by using a reactive forcefield (ReaxFF in the present work). Figure 8 shows the computed stress-strain curve when the crack is loaded in mode I. The crack propagation exhibits some small instabilities while the general behavior is a strain softening during failure. The underlying mechanism is a sequence of the brittle breaking of individual clay layers, similarly to the failure of layered composites with some delamination between the layers. This conclusion is supported by the evolution of the crack surface area upon loading. The increase of the crack surface area between stress drops 
indicates that small secondary cracks (delamination) are being created before breaking the next clay layer.

2 These secondary cracks merge upon further propagation of the crack.

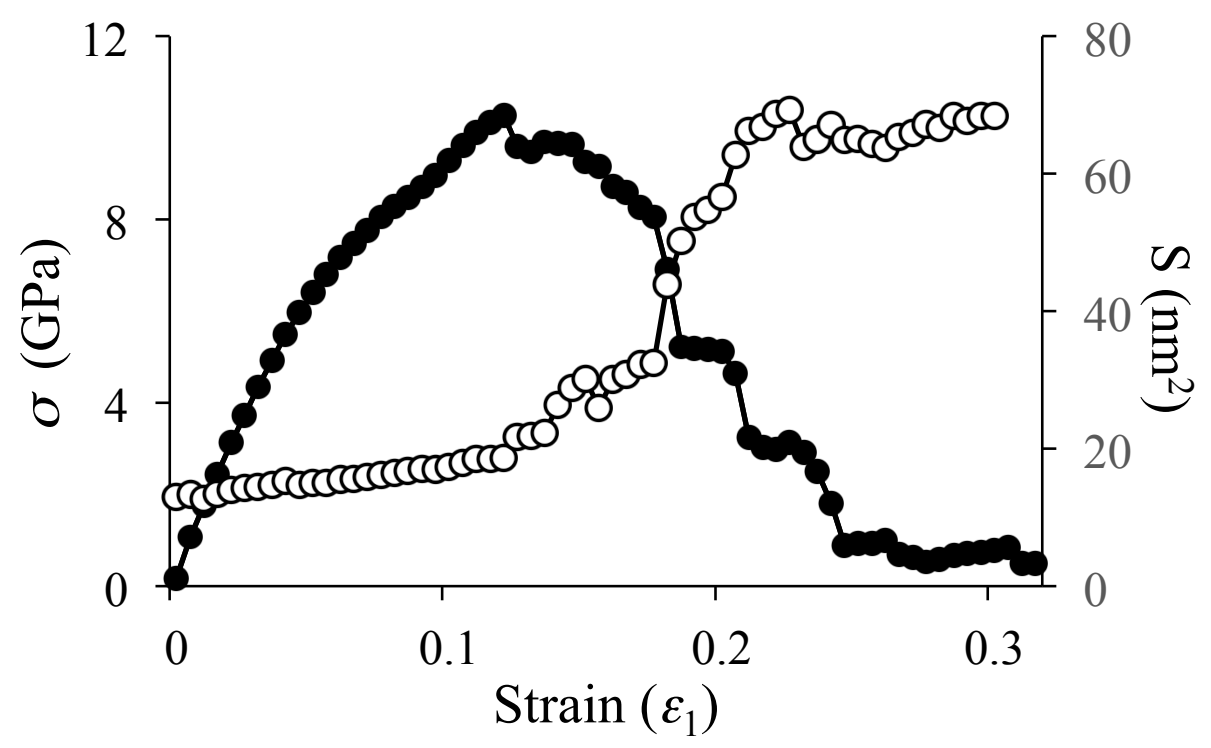

Figure 8.Stress $\sigma$ (filled circles, left axis) and crack surface area $S$ (empty circles, right axis) upon mode I loading of a crack perpendicular to the clay layers as a function of the imposed strain $\varepsilon_{\bullet}$. The typical molecular configurations show that the clay layers break in a sequential manner. The crack surface area is evaluated by the method described in Section 2.4. This curve reveals that the fracture propagation proceeds by first creating separate secondary cracks that eventually merge. After having evaluated the crack surface area created upon propagation of the crack in this direction, it is possible to compute the corresponding energy release rate $G_{C}$. Using Eq.(4) one obtains $G_{C}=3.86 \mathrm{~J} / \mathrm{m}^{2}$ which can also calculate from $G_{C}$ the corresponding fracture toughness value using an equation developed for a crack in this specific crack orientation (propagating along direction 3) and loaded in mode I. For TI materials, this expression reads: (Laubie, 2013)

$$
H_{11}=\frac{1}{2 \pi} \sqrt{\frac{C_{33}}{C_{11} C_{33}-C_{13}^{2}}\left(\frac{1}{C_{44}}+\frac{2}{C_{13}+\sqrt{C_{11} C_{33}}}\right)}
$$


2 For this crack orientation the fracture toughness was found to be $K_{C}=0.61 \mathrm{MPa} \cdot \mathrm{m}^{1 / 2}$. Table 4 compares the

3 fracture toughnesses obtained in this work for the different crack orientations. We also report experimental

4 value found in the literature for muscovite mica; (Spray, 2010) although somewhat different, this material is

5 often considered as a proxy for illite in terms of mechanical properties. Indeed, illite and muscovite differ

6 slightly in their number and distribution of isomorphic substitutions, (Bergaya, Then, \& Lagaly, 2006)

7 resulting in a charge of $-2 e$ for muscovite instead of $-1.5 e$ for illite. Both clay minerals contain, however, $\mathrm{K}^{+}$

8 counter ions only.

9

10 Table 4. Comparison of the calculated fracture toughness $K_{C}$ for illite with experimental data for muscovite

11 (Spray, 2010)
Simulation $\left(\mathrm{MPa} \cdot \mathrm{m}^{1 / 2}\right)$
Experimental $\left(\mathrm{MPa} \cdot \mathrm{m}^{1 / 2}\right)^{\mathrm{a}}$
(for illite)
(for muscovite mica)

$\begin{array}{lc}\text { Parallel to the } 001 \text { plane } & \text { Orthogonal to the } 001 \text { plane } \\ 0.09(\text { ReaxFF }) & 0.61(\text { ReaxFF }) \\ 0.12(\text { ClayFF }) & \end{array}$

${ }^{a}$ from (Spray, 2010)

14 Table 4 displays a consistent qualitative trend when comparing our calculated values to experimental data.

15 (We suppose that the same trend would be found when using the corresponding experimental value of illite.)

16 The trend shows that the experimental fracture toughness for muscovite is about twice the value calculated for

17 the 001 crack orientation in illite, but three times smaller than the computed value when the crack is

18 perpendicular to clay layers. To understand this result, one has to bear in mind that illite (and clays in general)

19 is a polycrystalline material at the macroscopic level and upscaling the simulated microscopic results to the

20 macroscale is not straightforward. In other words the representative elementary volume for the macroscopic

21 fracture properties is larger than what has been studied here. 
The direction of the fracture propagation is always determined by the lowest fracture resistance (fracture toughness) which is based locally on the grain orientation and the far field stress. As the crack can be subjected to various toughening phenomena when propagating in a granular material (such as crack bridging, crack deflection, front roughening, crack pinning, etc), the overall macroscopic fracture toughness will always be higher than the one in the preferred fracture propagation direction. Data in Table 4 suggest that fracture propagation is likely to happen in a direction parallel to the clay layers (001 direction) and will always be preferred over a direction perpendicular to them. Due to the possible toughening mechanisms mentioned above, it is consistent to find the measured macroscopic fracture toughness $\left(0.2 \mathrm{MPa} \cdot \mathrm{m}^{1 / 2}\right)$ higher than the value obtained in the possible preferred direction of the fracture propagation $\left(0.1 \mathrm{MPa} \cdot \mathrm{m}^{1 / 2}\right)$.

This work sheds light on the fracture properties of clays at the microscopic scale and, as such, can be regarded as the starting point for a mesoscopic fracture simulation and eventually to upscale fracture properties to the macroscopic level. This work is also the basis for investigation of more complex systems at the molecular scale, such as illite-organic composite materials. (Hantal, Brochard, Pellenq, Ulm, \& Coasne)

\section{Conclusions}

In this paper we studied the elastic and fracture properties of a typical clay mineral, illite, by means of molecular simulations. We used two force fields: the reactive ReaxFF potential which allows us to simulate bond breaking and formation during crack propagation and the non-reactive ClayFF potential which is known to accurately reproduce the structural properties of various clay minerals. The elasticity tensor of illite was determined using the two force fields and compared to that of a generic transversely isotropic material as an example of ideal layered materials.

The performance of the two force fields in terms of providing a reliable description of a transversely isotropic material was assessed based on three criteria: (i) the similarity of the calculated elasticity tensors to that of a transversely isotropic material; (ii) the distance of the elasticity tensors from that of an ideal TI material, and (iii) the magnitude of the error one would make by using the TI approximation to calculate fracture toughness 
instead of the general (less symmetric) formula. All these three comparisons indicate that ClayFF provides a

2 good description of the elastic properties of illite clay. In contrast, ReaxFF is found to fail at providing a

3 reliable description of the elastic properties. As a result, we decided to use the stiffness tensor determined with

4 ClayFF throughout the study to calculate fracture properties of illite. In contrast, fracture propagation

5 involving bond breaking and formation was studied using ReaxFF as it necessarily requires the use of reactive

6 forcefield.

8 Yield and fracture failures were also investigated. Failure in the 001 plane (i.e., the most likely cleavage 9 plane) was studied under tensile and shear loading, i.e. under mode I and mode II loading for cracked systems.

10 Various system and crack sizes were studied to make sure that our results are independent of the sizes of the 11 system and crack. In mode I, it was found that yield and fracture failures originate from a single mechanism: a 12 decohesion in the interlayer space characterized by an energy released per unit area of crack created, which is 13 the usual critical energy release rate in the case of fracture failure. The corresponding fracture toughness, 14 calculated with ReaxFF, was found to be quite low: $K_{c}^{\text {ReaxF }}=0.09 \mathrm{MPa} \cdot \mathrm{m}^{1 / 2}$. In contrast, in mode II loading, the yield failure mechanism is a stick-slip between clay layers. Fracture failure follows the same mechanism and no crack propagation could be observed. Again this is due to the lack of chemical bonding between the

17 clay layers so that they slide on top of each other. We repeated the simulation of the mode I loading of such a crack with the non-reactive ClayFF potential. We observed the same molecular mechanism, but we estimated a somewhat higher fracture toughness $K_{c}^{\text {ClayF }}=0.12 \mathrm{MPa} \cdot \mathrm{m}^{1 / 2}$. The mode I loading of a crack perpendicular to the clay layers was also investigated. We found that, in this case, the material exhibits a strain softening as the result of the clay layers breaking one after the other. Illite displayed a much higher fracture resistance $(0.61$

$\left.22 \mathrm{MPa} \cdot \mathrm{m}^{1 / 2}\right)$ in this orientation.

The highly anisotropic failure behavior of illite was explained as a result of its specific layered structure. The low failure resistance under tension and the stick-slip failure under shear are both the consequence of the lack of chemical bonds between clay layers where the cohesion is provided by electrostatic interactions only. The mode I fracture toughness values for the 001 orientation was found to be consistent with the experimental 
value $\left(0.2 \mathrm{MPa} \cdot \mathrm{m}^{1 / 2}\right)$ measured for muscovite, a clay mineral analogous to illite. The measured enhanced macroscopic fracture toughness value compared to our result was interpreted with toughening mechanisms arising from the granular structure of clays.

\section{Acknowledgement}

This work was funded by Royal Dutch Shell and Schlumberger in the framework of X-Shale research project.

We wish to thank A. Saul (CNRS/MIT) for helpful discussions.

$9 \quad$ References

10

Allen, M. P., \& Tildesley, D. J. (1989). Computer Simulation of Liquids. Oxford University Press.

Anderson, T. L. (2005). Fracture Mechanics: Fundamentals and applications. CRC Press.

Bergaya, F., Then, B. K., \& Lagaly, G. (2006). Handbook of clay science. Elsevier.

Bhattacharya, S., Coasne, B., Hung, F. R., \& Gubbins, K. E. (2009). Modeling Micelle-Templated Mesoporous Material SBA-15: Atomistic Model and Gas Adsorption Studies. Langmuir, 25, 5802-5813.

Botan, A., Marry, V., Rotenberg, B., Turq, P., \& Noetinger, B. J. (2013). J. Phys. Chem. C, 117, 978.

Britt, L. K., \& Schoeffler, J. (2009). The geomechanics of a shale play : What makes a shale prospective 1. SPE, 125525

Brochard, L., Hantal, G., Laubie, H., Pellenq, R., \& Ulm, F.-J. (n.d.). Fracture Toughness Calculation by Molecular Simulation: Cases of Silica, Microporous Carbons and their Interface. Submitted to J Mech Phys Solids.

Connolly, M. L. (1983). Analytical molecular surface calculation. J App/ Cryst , 16, 548-558.

Cygan, R. T., Liang, J. J., \& Kalinichev, A. G. (2004). J. Phys. Chem. B, 108, 1255-1266.

Ebrahimi, D., Pellenq, R. J.-M., \& Whittle, A. (2012). Langmuir, 28, 16855-16863.

Faust, J., \& Knittle, E. (1994). Geophys Res Lett, 21, 1935-1938.

Gale, J. D. (2002). GULP 3.1, General Utility Lattice Program.

Hantal, G., Brochard, L., Pellenq, R. J.-M., Ulm, F.-J., \& Coasne, B. (n.d.). Atomic scale simulation of the interfacial chemistry and failure properties of clay-organic composites. in preparation. 
8

Heinz, H. (2006). J. Chem. Phys., 124, 224713.

Hoenig, A. (1978). The behavior of a flat elliptical crack in an anisotropic elastic body. Int J Solids Struct, 14, 925-934.

Jorge, M., Jedlovszky, P., \& Cordeiro, M. N. (2010). A Critical Assessment of Methods for the Intrinsic Analysis of Liquid Interfaces. 1. Surface Site Distributions. J. Phys. Chem. C, 114, 20291.

Lammps: Molecular Dynamics Simulator, http://lammps.sandia.gov. (n.d.).

Laubie, H. U.-J. (2013). submitted to J. Eng. Mech, ASCE,.

Leblond, J.-B. (2003). La mécanique de la rupture fragile et ductile. Hermès.

Lee, B., \& Richards, F. M. (1971). The interpretation of protein structures: estimation of static accessibility. J Mol Biol, 55, 379-400.

Meunier, A., \& Velde, B. (2004). Illite.Origins, evolution and metamorphosis. Springer.

Militzer, B., Wenk, H. R., Stackhouse, S., \& Stixrude, L. (2011). Am Mineral, 96, 125.

Moakher, M. (2006). J. Elasticity, 82, 273.

Pitman, M. C., \& van Duin, A. C. (2012). J. Am. Chem. Soc., 134, 3042.

Refson, K., Park, S. H., \& Sposito, G. J. (2003). J. Phys. Chem. B, 107, 13376-13383.

Sato, H., Ono, K., Johnston, C. T., \& A., Y. (2005). Am Mineral, 90, 1824.

Sih, G. C., Paris, P. C., \& Irwin, G. R. (1964). International Journal of Fracture, 1, 189.

Spray, J. G. (2010). Annu. Rev. Earth Planet. Sci., 38, 221-254.

Teich-McGoldrick, S. L., Greathouse, J. A., \& Cygan, R. T. (2012). J. Phys. Chem. C, 116, 15099-15107.

Vaia, R. (2002). AMPTIAC Newsletter, 6, 17.

van Duin, A. C., Dasgupta, S., Lorant, F., \& Goddard, W. A. (2001). J. Phys. Chem. A. 105, 9396.

van Duin, A. C., Strachan, A., Stewman, S., Zhang, Q., Xu, X., \& Goddard, W. A. (2003). J. Phys. Chem. A, 107, 3803-3811.

Vandenbroucke, M., \& Largeau, C. (2007). Org Geochem, 38, 719-833.

Vanorio, T., Prasad, M., \& Nur, A. (2003). Geophys J Int, 155, 319-326.

Wang, J. W., Kalinichev, A. G., \& Kirkpatrick, R. J. (2006). Geochim Cosmochim Ac, 70, 562-582.

Zartman, G. D., Liu, H., Akdim, B., Pachter, R., \& Heinz, H. (2010). J. Phys. Chem. C, 114, 1763-1772. 